\title{
MAGNETIC MODELING VS MEASUREMENTS OF THE DIPOLES FOR THE JLAB 10 KW FREE ELECTRON LASER UPGRADE*
}

\author{
G. H. Biallas \#, K. Baggett, D.R. Douglas, T. Hiatt, R. Wines \\ Thomas Jefferson National Accelerator Facility, 12000 Jefferson Blvd. Newport News VA 23606 \\ T.J. Schultheiss, V.A. Christina, J.W. Rathke \\ Advanced Energy Systems, Inc. 27E Industrial Blvd., Medford, NY 11763 \\ A. Smirnov, D. Newsham, Y. Luo, D. Yu \\ DULY Research Inc. 1912 MacArthur Street, Rancho Palos Verdes, CA 90275-1111
}

\section{ABSTRACT}

Magnetic measurements of the six families of dipoles for the infrared Free Electron Laser Upgrade at the Thomas Jefferson National Accelerator Facility (Jlab) are compared to the magnetic models on which their design is based. The magnets were designed in parallel by three organizations. They used ANSYS, Radia or Opera 3D as a 3D magnetic modeling program. Comparison of the discrepancies between model and magnet measurement is presented along with analysis of their potential causes. These dipoles operate in two field ranges. The Injector/ Extractor Dipoles operate around $0.05 \mathrm{~T}$ and the Arc Dipoles and Optical Chicane Dipoles operate between 0.22 to $0.71 \mathrm{~T}$. All magnets are required to meet core field and field integral flatness to parts in $10^{4}$ over their "good field" region.

\section{INTRODUCTION}

Dipole magnets requiring core fields and field integrals to be uniform to parts in 10,000 require a marriage between magnetic modeling and final adjustment during magnet testing. This paper discusses how closely six families of dipoles matched their design models and the methods used to make the magnets usable.

Twenty-two dipoles are required for the injection, extraction, optical chicane and arcs (Table 1) of the $10 \mathrm{~kW}$ IR Free-Electron Laser Upgrade [1] at the Thomas Jefferson National Accelerator Facility (Jefferson Lab). The dipoles run at relatively low fields ranging from 0.05 to 0.7 Tesla. Two sets of $180^{\circ}$ "Bates Lab" arcs with three families of dipoles (designated GQ, GX, GY) form the arcs of the racetrack beam path. An additional family $(\mathrm{GW})$ of four dipoles transport the beam around one FEL resonator mirror. These four families operate between 0.16 and $0.71 \mathrm{~T}$. Two low field $(0.05 \mathrm{~T})$ families $(\mathrm{GU}$, $\mathrm{GV})$ reside in the injection and extraction lines.

We used the window frame with Purcell gap system dipole style of the IR Demonstration FEL [2] as the starting point for designs. We modeled the dipoles using computer models making use of faster computers and smaller grids than had been used in the past to resolve fields in $3 \mathrm{D}$ to the part in $10^{5}$ level.

Table 1: Dipole Family Characteristics

\begin{tabular}{|c|c|c|c|}
\hline $\begin{array}{c}\text { Designator } \\
\text {-Use }\end{array}$ & Qty. & $\begin{array}{c}\text { Effective } \\
\text { Length }\end{array}$ & Core Field \\
\hline $\begin{array}{c}\text { GV - Injection/ } \\
\text { Extraction }\end{array}$ & 6 & 0.21 & $0.045-0.061$ \\
\hline $\begin{array}{c}\text { GU - Injection/ } \\
\text { Extraction }\end{array}$ & 2 & 0.43 & $0.045-0.061$ \\
\hline $\begin{array}{c}\text { GW - Optical } \\
\text { Chicane }\end{array}$ & 4 & 0.42 & $0.223-0.592$ \\
\hline GX - Arc Bend & 4 & 0.894 & $0.222-0.584$ \\
\hline $\begin{array}{c}\text { GQ- Arc Reverse } \\
\text { Bend }\end{array}$ & 4 & 0.917 & $0.222-0.584$ \\
\hline $\begin{array}{c}\text { GY- Arc Pi }\left(180^{\circ}\right) \\
\text { Bend }\end{array}$ & 2 & 3.14 & $0.267-0.711$ \\
\hline
\end{tabular}

We took care to build the magnets to match the magnetic models. The primary item to match is precision gap. We obtained gap uniformities at the part in 10,000 level $(7 \mu \mathrm{m})$. This tolerance is at the limit of achievement at skilled machine shops by machining or grinding). A gapmeasuring instrument, using a Starrett distance measuring instrument, with $0.25 \mu \mathrm{m}$ resolution and electronic read-out enabled this difficult measurement.

*Work supported by the US DOE Contract \#DE-AC0584ER40150, the Office of Naval Research, The Air Force Research Laboratory, the Commonwealth of Virginia, and the Laser Processing Consortium.

\# Email: biallas@jlab.org 
The next item we attempted to match to the model is the iron in the core. The magnet cores are made of solid 1006 steel while all field clamps and some auxiliary core parts of the GYs are made of 1018 steel. Families of magnets were made of 1006 steel from the same melt. All 1006 had solution annealing in vacuum at $965 \mathrm{C}$ before final machining. All manufacturers were cautioned to not leave magnetic footprints on pole surfaces from lifting or chucking magnets. Finally, all magnets were run on hysteresis loops at the operations ramp rate to a maximum current above the set point and then set to running current in order to standardize residual fields.

All magnets have field clamps that minimize stray field. Field clamp adjustability in the beam direction also provides ability to adjust the effective length by about $0.5 \%$.

The core field and field integral tolerances for these magnets are the same as the corresponding dipoles of the IR Demo and are repeated in detail elsewhere at this conference. [3] Basically, core fields and field integrals are to match to 1 part in 1000 within a family and core field and integral for any one magnet should be uniform within a few parts in 10,000 over the good field region. All but one family met these tight magnetic specifications over their required field ranges. (See section 5 for an explanation of the exception) Two families matched the models beautifully. The remainder required one or more adjustments at the Magnet Measurement Facility at Jefferson Lab. [3,6] The injection and extraction dipoles were accepted for a single momentum of $9.2 \mathrm{MeV} / \mathrm{c}$. The recirculation magnets were characterized at core field levels corresponding to 80,145 and $210 \mathrm{MeV} / \mathrm{c}$ with $145 \mathrm{MeV} / \mathrm{c}$ as the baseline.

\section{INJECTION AND EXTRACTION DIPOLES (GU/GV)}

DULY Research designed the two families of window frame style injection and extraction dipoles [4] using the 3D program Radia. The dipoles were designed at a maximum field of $0.061 \mathrm{~T}, 10 \%$ over the field required for nominal $10 \mathrm{MeV} / \mathrm{c}$ operation.

The small dipole $(\mathrm{GV})$ was the most difficult magnet to model of all dipoles because it is short, has a large gap in relation to its length and is a $20^{\circ}$ wedge. At the narrow part of the good field region, the magnet length is the same as the gap. Characteristic end field fall-offs within the gap from both ends of the magnet superpose. The resulting droop in the core field on the narrow side makes it difficult to obtain field flatness to parts in 10,000 over the good field region. DULY found that using the mu metal Purcell gap system [2] was not effective for flattening the field. DULY Research invented a trim coil system as a solution to this problem. DULY also incorporated a method to adjust out the nonlinear field integral gradient across the good field region seen in their models.

The large injection/ extraction dipole (GU) is nominally double the magnetic length of the GV and much simpler. The GU was able to use the Purcell gap system developed for the IR Demo to flatten the core field and didn't require the trim coil.

Results: DULY's trim coil system worked to make the core field flat over the good field region. However, the current is $7 \%$ higher than predicted. In addition, we found that the magnetic wedge angle (field integral gradient) of this $20^{\circ}$ wedge magnet is not constant with excitation. Therefore we picked the momentum the injector was set at in the IR Demo (9.2 $\mathrm{MeV} / \mathrm{c}$ ) as a single operating point. We adjusted the angle of the field clamps to bring the integral gradient to a $20^{\circ}$ wedge at the core field for this momentum. Additional measurements provided the field clamp positions for other excitations. The field integrals were smaller than predicted. Since the magnet is a wedge and we had excess width to the good field region, we shifted the wider side of the magnet transverse to the beam centerline to obtain the specified field integral on beam centerline. We found that magnetic shim material on the inside of the field clamps on either side of beam centerline corrected a problem of field integral gradient nonlinearity. The magnet was ramp rate sensitive so all testing was done at ramp rate used for operations .

The modeling of this low field magnet where much of its core is running in saturation was particularly challenging in view of the high fringe fields and non-uniformity for magnetic 
flux distribution [4]. Using the adjustable trim coils together with the adjustments and accommodations discussed earlier, this magnet family has the best conformance to specification.

Results on the GU magnet showed that the model was correct in its uniformity predictions but the field integral was $2 \%$ long. We shortened the pole tips by machining the end faces.

Since the GVs and GU were to be run on the same power supply, we added a variable shunt around the GU magnet that permits matching the magnets.

\section{OPTICAL CHICANE DIPOLE GW}

The Jlab Engineering Group modeled the GW dipole as a window frame style rectangular pole magnet with Purcell gap system using Opera 3D. All magnet values measured as predicted, matching the GX/GQ magnets.

\section{BEND/REVERSE BEND DIPOLES GX/GQ}

Advanced Energy Systems Corp. (AES) modeled the GX \& GQ magnets using ANSYS. [5] Micrometric steps in the pole tips at the ends and corners shaped the fields to meet uniformity requirements. The magnets are " $\mathrm{H}$ " style to allow optical pipes through the return legs of the yokes.

The GX has a large wedge angle $\left(39.4^{\circ}\right)$ and a small good field region $(10.2 \mathrm{~cm})$. The pole is enlarged in one region for a second beam path at half field. When half the coil turns are powered, the magnet is a switch, sending beam to the UV branch. The Reverse Bend Magnet (GQ) has a small wedge angle $\left(10.4^{\circ}\right)$ with larger good field region $(25.4 \mathrm{~cm})$. Both families are similar enough to be powered in series with no shunt.

Results: The GQ core field and field integral uniformity at 145 and $210 \mathrm{MeV} / \mathrm{c}$ matched the model's profile. Field uniformity at $80 \mathrm{MeV} / \mathrm{c}$ operation was only obtained by using a reduced current hysteresis loop. In addition, field integral was $0.91 \%$ short. We rectified this by adding magnetic shims to lengthen the poles. The field integral gradient was not linear near the centerline. We rectified this by adding $0.75 \mathrm{~mm}$ shims to the inside center of the field clamps. The Bend Magnet (GX) matched the model (including the half field "switch" mode) well enough in all respects to meet specification.

\section{PI $\left(180^{\circ}\right)$ BEND GY}

AES designed the Pi Bend as a window frame style with Purcell gap system using ANSYS. [5]. Modeling indicated factors of 3 to 4 improvement in field uniformity with the system. The magnet has additional coils built into either end of its poles, making the pole portions into path length correctors (designated GG coils).

Results: Unlike the model, the first magnet exhibits quadrupole at the part in 1000 level and sextupole at the parts in 10,000 level. [6] (Perhaps due to an over-current powering at the start of measurement which we never cancelled by degaussing.) We will compensate for these errors using adjacent multipole trim magnets. Field integral was short by $0.61 \%$. We rectified this by adding $1 \mathrm{~cm}$ shims to the pole end faces.

\section{SUMMARY AND CONCLUSIONS}

Three $\mathrm{D}$ modeling and test resulted in dipoles that qualified for installation. Modeling provided excellent design guidance for obtaining field uniformity as well as far more accurate effective lengths than obtainable with $2 \mathrm{D}$ modeling. The GW and GX families qualified directly. The GQ and GY ANSYS models predicted effective lengths within $1 \%$ at intermediate fields. For low field magnets, Radia's length prediction was $2 \%$ high for the GU and its current prediction for the highly saturated GV was 7\% low. All out-oftolerance conditions were recoverable using adjustments, shims, pole end machining, shunts and (as a last resort) corrector magnets. We had provisions for all of these measures built into the magnets or available to us.

\section{REFERENCES}

[1] D. Douglas, et.al. "A $10 \mathrm{~kW}$ IRFEL Design For Jefferson Lab", Proc. PAC2001, Chicago, June 2001.

[2] G, Biallas, et.al. " Making Dipoles to Spectrometer Quality Using Adjustments During Measurement ", Proc. PAC1999, New York, (1999).

[3] T. Hiatt, et.al. "Magnetic Measurement of the $10 \mathrm{~kW}$, IR FEL Dipole Magnets". Proc. PAC2003, Portland, (2003).

[4] D. Newsham, et. al. "Wedge-shaped, Large-aperture, Dipole Magnet Designs for the Jefferson lab Facility Upgrade", Proc. PAC2003, Portland, (2003).

[5] T. Schultheiss, et al. "Magnetic Analysis of the Arc Dipoles for the JLAB $10 \mathrm{~kW}$ Free Electron Laser Upgrade", FEL'02, Argonne, IL, Sep. 2002.

[6] K Baggett, et.al., "Magnetic Measurement of the $10 \mathrm{~kW}$, IR FEL 180 Degree Dipole”. Proc. PAC2003, Portland, (2003). 
